\title{
Correlations between dose, plasma concentrations, and antispastic action of tizanidine $\left(\right.$ Sirdalud ${ }^{\circledR}$ )
}

\author{
M Emre, G C Leslie, C Muir, N J Part, R Pokorny, R C Roberts
}

\begin{abstract}
In a double blind, placebo controlled, cross over study the correlations between single doses $(2,4$, and $8 \mathrm{mg})$, plasma concentrations, and antispastic action of tizanidine were investigated in 16 patients with extensor spasticity of the legs due to multiple sclerosis. An electrogoniometer was used to assess muscle tone at knee extensors, applying Wartenberg's pendulum test. Blood samples, a clinical assessment of muscle tone by the Ashworth scale, and muscle strength by the British Medical Research Council scale were obtained concomitantly. Confirmatory analysis using the change in the relaxation index ( $R 2$ value) $1 \cdot 5$ hours after each treatment, showed a statistically significant $(p=0.0123)$ linear dose-response relation between single doses and antispastic action of tizanidine. Further statistical analysis showed a strong within patient linear correlation between plasma concentrations and antispastic action at 4 and $8 \mathrm{mg}$ doses $(p=$ 0.014 and 0.004 respectively), but only weak between patient correlations. The analysis of the dose-plasma concentration relation showed results consistent with linear pharmacokinetics. The comparison of changes in the $R 2$ ratio with concomitant Ashworth scores showed a significant correlation between the two. It is concluded that there are linear correlations between single doses, plasma concentrations, and antispastic action of tizanidine. Because of the strong within patient but weak between patient correlation between plasma concentrations and antispastic action of tizanidine the effective doses should be determined individually.
\end{abstract}

(F Neurol Neurosurg Psychiatry 1994;57:1355-1359)

Tizanidine (Sirdalud ${ }^{\circledR}$ ) is a centrally active $a_{2}$ adrenergic receptor agonist with potent myotonolytic action. ${ }^{1-4}$ The substance was shown to suppress selectively polysynaptic spinal reflexes while sparing the monosynaptic reflexes. ${ }^{5}$ Clinical investigations have consistently shown an antispastic effect of tizanidine that was associated with little undue muscle weakness, ${ }^{6-9}$ sometimes associated with an improvement in paresis. ${ }^{10}$ There is, however, little information on the correlation between the antispastic effect and plasma concentra- tions of tizanidine. ${ }^{11}$ We report data on the correlations between single doses, plasma concentrations, and antispastic action of tizanidine in patients with spasticity due to multiple sclerosis, by means of an objective quantitative method and a clinical scale for the assessment of spasticity.

\section{Methods}

\section{PATIENT SELECTION}

Seventeen patients were entered into the study, but one patient was excluded from further analysis because the baseline severity of spasticity decreased during the study to below that stipulated in the protocol. There were 13 women and four men, mean age 43 (range 24-58) years. All patients had definite multiple sclerosis, diagnosed by clinical criteria and paraclinical investigations. Spasticity had been present for a mean of 70 (range 12-180) months and had been stable for a mean of 17 (range 2-48) months. Extensor spasticity reaching a minimum score of 2 on the Ashworth scale in at least one leg and remaining stable for at least one month was required as an entry criterion. All patients had a baseline score of either 2 or 3 on knee extensors, none had a score of 4 , and none of them were bedridden. Patients receiving any drugs with antispastic action, those with significant systemic diseases, local complications, an exacerbation of the disease, or abnormalities in laboratory tests were excluded from the study.

\section{EXPERIMENTAL PROCEDURE}

The protocol was approved by the committee on medical ethics of Dundee General Hospitals, and informed consent was obtained from each patient before they entered the trial. Patients were allocated a treatment sequence according to a randomisation schedule based on a latin square design. Each patient received in a double blind, randomised, cross over design single doses of 2 $\mathrm{mg}, 4 \mathrm{mg}$ and $8 \mathrm{mg}$ of tizanidine and one dose of placebo on four separate days, allowing a wash out period of at least 72 hours between the different doses. Tizanidine and placebo tablets had the same appearance, and each dose contained the same number of tablets with the same appearance to maintain blindness.

The examination schedule and content as well as timing of meals were standardised for each patient and across the patients. The medication was taken with a glass of water 
between 800 and 900 am, 30 minutes before breakfast and after the baseline evaluations had been performed. An indwelling catheter was placed in the cubital vein 30 minutes before the daily baseline evaluations. Blood samples were taken at baseline, 15, and 30 minutes and $1,1 \cdot 5,2,2 \cdot 5,3,4,6$, and 8 hours after dosing. Plasma tizanidine concentrations were analysed by a specific radioimmunoassay method with a sensitivity of $250 \mathrm{ng} / 1$.

\section{MEASUREMENT OF SKELETAL MUSCLE TONE} Each patient was given an initial screening assessment at which muscle tone was evaluated for both legs. Thereafter all measurements were performed on the leg that had the higher spasticity score at the knee extensors. The tone of the quadriceps muscle was measured by means of an electrogoniometer and applying the Wartenberg pendulum test. ${ }^{12}$ The principle of this test is that the swing pattern of the lower leg, as it swings under the action of gravity, depends on the tone in the quadriceps muscle. A quantified version of the test was reintroduced by Bajd and Vodovnik $^{13}$ and is able to distinguish spasticity and rigidity. ${ }^{14}$ The following procedure was used for taking a measurement: The patients lay on a couch with the lower legs hanging over the end. The examined leg was indirectly attached to an electrogoniometer (Penny and Giles, Type PGS) so as to measure the angle at the knee by the method described by Bajd and Vodovnik. ${ }^{13}$ The lower leg was lifted as near to the horizontal as possible without taking the upper leg off the couch. Having ensured that the leg was relaxed, the lower leg was then released and allowed to swing freely under gravity. The angle data were sampled for 10 seconds by a 286 PC microcomputer

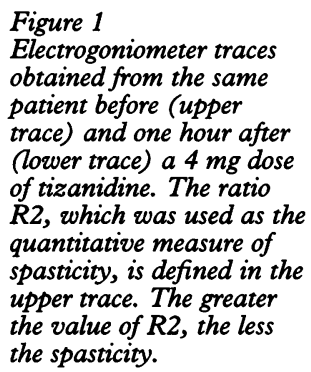

via a CED 1401 intelligent interface (Cambridge Electronic Design). Seven tests were taken at each assessment session. The first swing was for practice and it was not included in the analysis. Statistics of the assessment data were obtained by calculating the mean value of each variable from the next six tests. During the examination patients were distracted by music played through headphones to maximise muscle relaxation and to standardise experimental conditions. Goniometric assessments were performed at baseline immediately before drug intake and 30 minutes, $1,1 \cdot 5,2,3,4,6$, and 8 hours after the drug intake.

Bajd and Vodovnik ${ }^{13}$ have proposed that the best quantitative assessment of spasticity is made from the relaxation index, $\mathrm{R} 2$, and this has been confirmed by Brown et al. ${ }^{14}$ This is the ratio of the amplitude of the first swing of the leg on release to the difference in angle between the start and final resting position (fig 1). The relaxation index R2 was calculated with PC software. The log of the ratio R2 was used for the confirmatory data analysis. Further data were derived from the pendulum swing traces for descriptive and exploratory analysis. The additional measurements were the ratio $\mathrm{R} 1$ (the ratio of the amplitude of the first swing to the amplitude of first rebound swing), the maximum velocity on the first swing, the maximum velocity on the first rebound swing, the maximum acceleration on the first swing, and the maximum acceleration on the rebound swing.

Muscle tone was also assessed clinically with the Ashworth scale. ${ }^{15}$ Tone of both the knee extensors and flexors was assessed immediately after each series of goniometric measurements. The strength of knee flexor and extensor muscles was also assessed with the British Medical Research Council (BMRC) scale. The systolic and diastolic blood pressures and pulse rate were measured after each goniometric assessment in each session. Any adverse event occurring after drug intake was recorded.

DATA ANALYSIS

The concept described by $\mathrm{Abt}^{16}$ was used to analyse and interpret the data. Three types of statistical analysis were performed, which were predetermined in the study protocol.

\section{Confirmatory data analysis}

This analysis was performed to assess the linearity of the dose-effect relation at the expected time of maximum effects ( 1.5 hours after medication), with the $\log _{10}$ transformed value of the $R 2$ ratio. The individual effects of the four treatment regimens (placebo, $2 \mathrm{mg}, 4$ $\mathrm{mg}$, and $8 \mathrm{mg}$ of tizanidine) were split into a linear, a quadratic, and a cubic component. These were tested for statistical significance including also the sequence effect (one way analysis of variance (ANOVA), $p=0.05$ ). The dose was transformed as $\log \left(1+\mathrm{d}_{\mathrm{i}} / \mathrm{d}_{1}\right)$, where $d_{i}$ was 0 for $d_{o}$ (placebo), 2 for $d_{1}(2$ $\mathrm{mg}), 4$ for $\mathrm{d}_{2}(4 \mathrm{mg})$, and 8 for $\mathrm{d}_{3}(8 \mathrm{mg}$ of tizanidine). 
Figure 2 Dose efficacy relation in the $2-8 \mathrm{mg}$ dose range of tizanidine. The $y$ axis represents $\log R 2$ value 1.5 hours after each dose of tizanidine and placebo. The $x$ axis represents the dose expressed as log (1+ dose/2). The slope of the linear trend component has $a$ value of $0.0314(p=$ 0.0123 ).

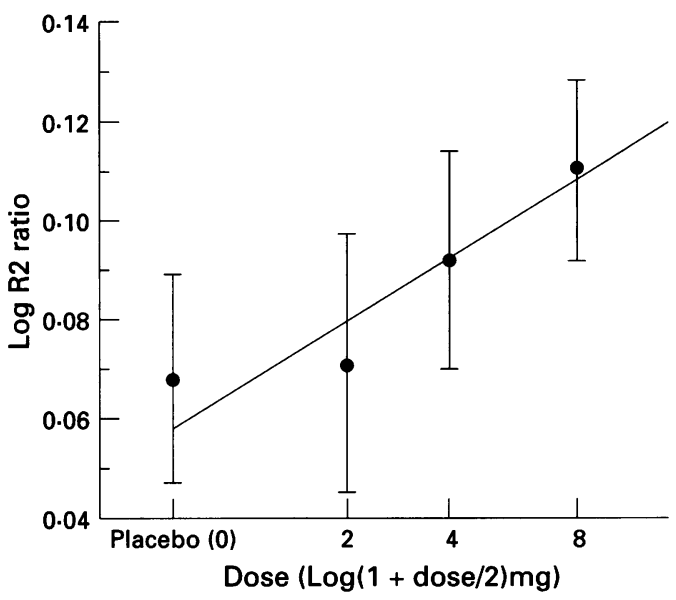

Descriptive data analysis

All goniometric variables and clinical scores obtained after each dose of study medication were compared with those obtained after placebo, at each time point after medication. The evaluations were performed with both actual values and baseline corrected values (differences from baseline on the day of experiment) by means of two way ANOVA for goniometric variables and the Wilcoxon matched pair signed rank test for the clinical scores $(p=0.05)$.

Exploratory data analysis

In this analysis the linear correlations between plasma concentrations of tizanidine and goniometric variables (difference from placebo in the baseline corrected values) were investigated. Within patient empirical correlation coefficients were tested for difference from zero for each dose, as well as between patient empirical correlation coefficients for each time point and each dose. In addition, dose normalised area under the plasma concentration-time curve (AUC 0-8 hours) and maximum plasma concentrations $\left(\mathrm{C}_{\max }\right)$

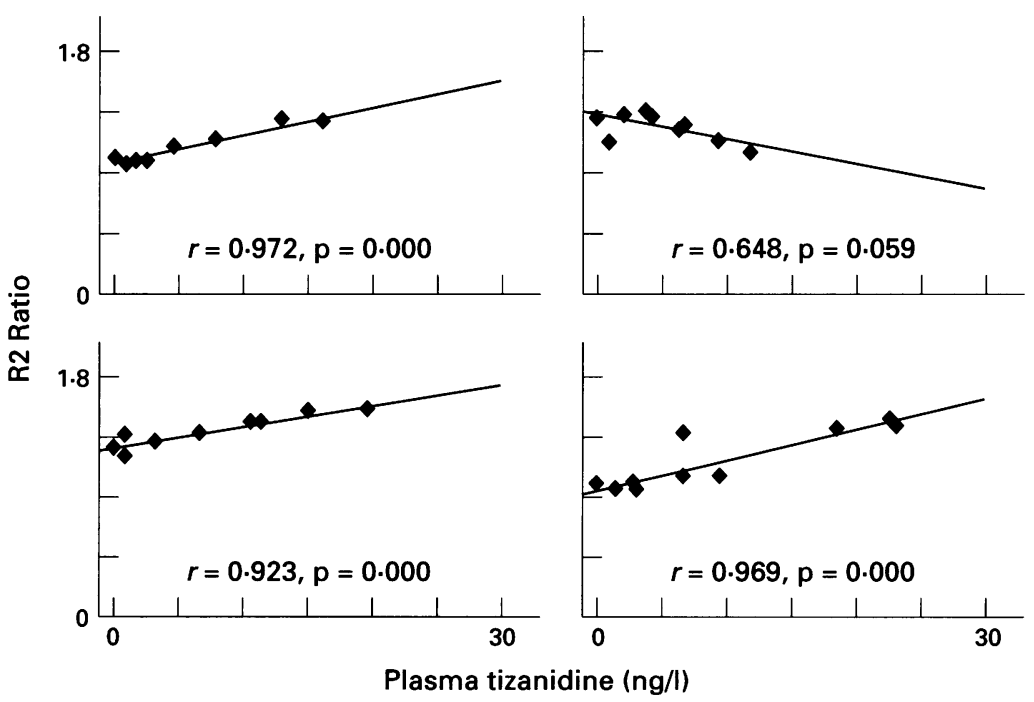

Figure 3 Ratio R2 (see fig 1) v plasma concentrations of tizinadine for four patients. For each subject the data were obtained on the day on which the $8 \mathrm{mg}$ dose of tizinadine was each subject the data were obtained on the day on which the $8 \mathrm{mg}$ dose of tizinadine was
given. On each graph the value of the correlation coefficient $(r)$ is given, as is the level of significance $(p)$ of the difference in slope of the line from zero. were compared by means of a two way ANOVA $(p=0.05)$, and $R 2$ values were compared with the Ashworth score (difference from placebo) by a within patient rank correlation analysis.

\section{Results}

DOSE-RESPONSE RELATION

Figure 1 gives examples of goniometric recordings before and after tizanidine. In the confirmatory analysis the $\log _{10}$ of the difference between the $R 2$ ratio at 1.5 hours and the $R 2$ ratio at baseline for that day and a log transformation of the dose was examined by regression analysis ( $F$ test). This analysis gave $p=0.0123$ for the linear component of the dose-response curve, indicating a statistically significant relation between the dose and antispastic action of tizanidine in the single dose range of 2 to $8 \mathrm{mg}, 1.5$ hours after medication. As a $\log$ transformation was used for both the dose and the $\mathrm{R} 2$ ratio, the doseresponse relation was considered to be linear (fig 2). There were no non-linear components to this relation, as the quadratic and cubic components of the dose-response curve were not statistically significant $(p=0.413$ and 0.708 respectively). In the descriptive data analysis antispastic effects different from that seen under placebo occurred up to three hours after taking the drug.

Analysis of variance for treatment/period interactions showed no evidence for carry over effects between the two sequences of placebo followed by $8 \mathrm{mg} v 8 \mathrm{mg}$ followed by placebo.

PLASMA CONCENTRATION-RESPONSE RELATION In each patient, linear correlations were calculated for each dose between plasma concentrations and the goniometric variables. Figure 3 gives examples of regression lines between the plasma concentration of tizanidine and concomitant $\mathrm{R} 2$ ratios at the $8 \mathrm{mg}$ dose for four patients. For nine of the 16 patients the slope of the regression line was significantly different from zero. Overall within patient correlation analysis between plasma concentration and the difference in the $\mathrm{R} 2$ ratios of placebo and active treatment gave a mean correlation coefficient of $0.005(p=0.967)$ for the $2 \mathrm{mg}$ dose, $0.347(\mathrm{p}=0.014)$ for the $4 \mathrm{mg}$ dose, and $0.536(\mathrm{p}=0.004)$ for the $8 \mathrm{mg}$ dose.

As well as the analysis of within patient correlations, between patient linear correlations between plasma concentrations and the difference in $\mathrm{R} 2$ ratios between placebo $v$ active treatment were also calculated. The analysis showed that 14 out of $288(4.9 \%)$ calculated correlations were significant. No $p$ pattern (clustering of significant correlations at a dose, time point, or both) with regard to time after drug treatment or dose dependency of significant locations was found, suggesting that "nominal" significances occurred by chance. This indicated a rather weak relation across subjects between the antispastic effects of tizanidine and plasma concentrations. 
DOSE-PLASMA CONCENTRATION RELATION

Plasma concentrations of tizanidine were obtained at 11 standard times in each investigation day. Figure 4 shows the mean drug plasma concentration curves at the three doses. In the exploratory data analysis, the statistical evaluation of dose-normalised area under the curve (AUC) and the maximum concentration $\left(C_{\max }\right)$ at the three dose levels examined gave results consistent with linear pharmacokinetics in the dose range used. The variability in the peak plasma concentrations between patients given the same dose of tizanidine was low ( $36 \%$ at $2 \mathrm{mg}, 29 \%$ at 4 and $8 \mathrm{mg}$ ).

\section{CORRELATION BETWEEN ASHWORTH SCORE AND R2 RATIO}

The mean change from baseline in Ashworth score two hours after medication was $28 \%$ for placebo, $29 \%$ for $2 \mathrm{mg}, 34 \%$ for $4 \mathrm{mg}$, and $38 \%$ for $8 \mathrm{mg}$. These mean changes, however, must be interpreted with caution given the non-linear nature of the Ashworth scale. The comparison of changes in the $\mathrm{R} 2$ value with concomitant changes in the Ashworth score for extensor muscles by means of a within patient rank correlation analysis showed significant correlations between the two variables at all doses, indicating a strong within patient linear correlation between the $\mathrm{R} 2$ ratio and Ashworth score for extensor tone (Spearman rank order correlation coefficients $0.327 \mathrm{(p}=$ $0.01)$ for $2 \mathrm{mg} ; 0.431(\mathrm{p}=0.009)$ for $4 \mathrm{mg}$, and $0.629(\mathrm{p}=0.0001)$ for $8 \mathrm{mg}$ ). Similarly, a between patient rank correlation analysis showed that the changes in these two variables significantly correlated with each other at all dose levels. The number of significant correlations increased dose dependently. The R2

Figure 4 Mean concentration-time curves for 16 patients. The three different doses of the drug were given on different days.

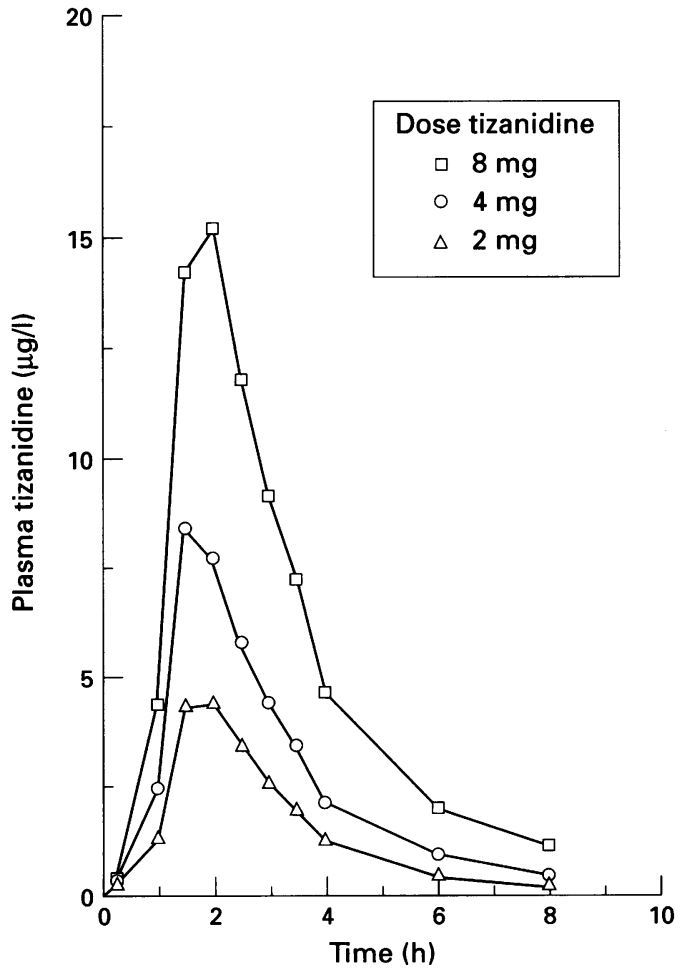

ratio and Ashworth score were negatively correlated in both between and within patient correlation analysis - that is, the higher the R2 ratio, the lower the extensor tone on the Ashworth scale.

Muscle strength, as measured by the BMRC scale did not show any detectable decrease in either extensor or flexor muscle strength at any time after any of the doses of tizanidine.

There was a mild decrease in blood pressure (mean $18 \mathrm{~mm} \mathrm{Hg}$ systolic and $10 \mathrm{~mm} \mathrm{Hg}$ diastolic pressure) and heart rate (5 beats $/ \mathrm{min}$ ), one to two hours after the $8 \mathrm{mg}$ single dose of tizanidine. Adverse events appeared in a dose dependent manner. Drowsiness was the most frequent adverse event (one patient in the $2 \mathrm{mg}$, four patients in the $4 \mathrm{mg}$, nine patients in the $8 \mathrm{mg}$, and three patients in the placebo group), followed by dry mouth. All adverse events were rated mild to moderate, and there were no severe adverse events. Total number of adverse events as well as the sum of intensity scores peaked around two hours after drug administration, roughly corresponding to the time of maximum plasma concentration.

\section{Discussion}

The purpose of this study was to assess the relations between dose, plasma concentrations, and antispastic effects of tizanidine. Spasticity was assessed by an electrogoniometer using Wartenberg's pendulum test, which gives an objective, quantitative assessment of knee extensor muscle tone. On the basis of previous experience ${ }^{14}$ the ratio $\mathrm{R} 2$ was chosen as the primary outcome measure of efficacy. Confirmatory analysis showed a linear relation between dose and antispastic efficacy of tizanidine in the single dose range of $2 \mathrm{mg}$ to $8 \mathrm{mg}, 1.5$ hours after drug intake; this was roughly at the time at which the peak plasma concentrations of the drug occurred. In two other studies, the antispastic action of tizanidine was maximum also at 60-90 minutes. ${ }^{11} 17$

Within patient correlation analysis of the relation between plasma concentrations of tizanidine and its antispastic effect showed a strong linear relation at the two higher doses. There was also a linear relation between dose and plasma concentration. Thus at the single doses examined there were strong within patient correlations between dose, plasma concentrations, and antispastic efficacy of tizanidine. No clearcut linear correlation, however, was found in the between patient analysis of the relation between plasma concentrations and antispastic effects of tizanidine. These results suggest that at a given plasma concentration the antispastic action of tizanidine may vary from patient to patient. Therefore, to achieve sufficient antispastic efficacy in a given patient, individual effective plasma concentrations should be reached and maintained. As suggested by the weak between patient correlations, the effective unit doses can vary from patient to patient. The effects of the $2 \mathrm{mg}$ dose of tizanidine on 
spastic muscle tone did not differ from placebo, so that unit doses higher than $2 \mathrm{mg}$ are likely to be necessary in most patients.

This study also showed a good correlation between the clinical scale (Ashworth scale) most often used to assess spasticity and the objective, quantitative measurement of muscle tone by an electrogoniometer. This finding is important as it suggests that used in a standard way (by the same investigator), the Ashworth scale can be reliably used to assess spasticity in large scale clinical studies in which the use of an electrogoniometer may not be possible. Electrogoniometric measurements, however, seemed to be more sensitive than the clinical assessment to the changes in muscle tone, so that small changes may not be detected by the Ashworth scale. The results also suggest that the electrogoniometer can be reliably used to provide quantitative assessment of muscle tone in spasticity.

At doses at which there was a clinically measurable decrease in spastic muscle tone with the Ashworth scale, no clinically detectable decrease in muscle strength was found on the BMRC scale. This suggests that the antispastic action of tizanidine is not associated with any undue muscle weakness. In fact, in some patients an antiparetic effect was associated with the antispastic effect of tizanidine. ${ }^{10}$ It must be borne in mind, however, that the BMRC scale may not be sensitive enough to measure slight changes in muscle strength.

Single doses of tizanidine up to $8 \mathrm{mg}$ were well tolerated. The adverse event profile did not differ from that known from previous experience. ${ }^{9}$ The most frequent adverse event was mild drowsiness. The frequency and severity of adverse events increased in a roughly dose dependent manner and peaked around the time of maximum plasma concentration. This suggests that adverse events may be related to peak plasma concentrations.

It is concluded that there is a linear relation between single doses, plasma concentrations, and antispastic effects of tizanidine in the 2-8 $\mathrm{mg}$ dose range. This relation is especially strong in within patient comparisons-that is, in a given patient the antispastic effect is closely related to plasma concentrations of tizanidine. The between patient correlations between plasma concentrations and antispastic effects seem to be less pronounced; thus a certain plasma concentration does not always lead to the same amount of muscle relaxation in all patients. Because a close within patient relation between dose, plasma concentration, and efficacy was found, the effective doses of tizanidine should be determined individually for each patient. The unit doses and dose intervals should be chosen so as to maintain an effective plasma concentration.

1 Sayers AC, Bürki HR, Eichenberger E. The pharmacology of 5-chloro-4-(2-imidazolin-2-yl-amino)-2, 1, 3-benzothiadiazole (DS 103-282), a novel myotonolytic agent. Arzneimittelforschung 1980;30:793-803.

2 Davies J. Selective depression of synaptic transmission of spinal interneurons in the cat by a new centrally acting spinal interneurons in the cat by a new centrally acting
muscle relaxant, DS 103-282. Br f Pharmacol 1982;76: muscle re

3 Curtis DR, Leah JD, Preet MJ. Spinal interneuron depression by DS 103-282 Brf Pharmacol 1983;79:9-11.

4 Davies J, Johnston SE, Hill DR, Quinlan JE. Tizanidine (DS 103-282), a centrally acting muscle relaxant, selectively depresses excitation of feline dorsal horn neurons to noxious peripheral stimuli by an action at alpha-2adrenoceptors. Neurosci Lett 1984;48:197-202.

5 Coward DM. Selective muscle relaxant properties of tizanidine and an examination of its mode of action. Triangle 1981;20:151-8.

6 Lapierre Y, Bouchard S, Tansey C, Gendron D, Barkas WJ, Francis GS. Treatment of spasticity with tizanidine in multiple sclerosis. Can $\mathcal{F}$ Neurol Sci 1987;14:513-7.

7 Eyssette M, Rohmer F, Serratrice G, Warter JM, Boisson D. Multi-centre, double-blind trial of a novel antispastic agent, tizanidine, in spasticity associated with multiple sclerosis. Curr Med Res Opin 1988;10:699-708.

8 Hoogstraten MC, van der Ploeg RJO, van der Burg W, Vreeling A, van Marle S, Minderhoud JM. Tizanidine versus baclofen in the treatment of spasticity in multiple sclerosis patients. Acta Neurol Scand 1988;77:224-30

9 Emre M. Review of clinical trials with tizanidine (Sirdalud ${ }^{\star}$ ) in spasticity. In: Emre $M$ and Benecke $R$, eds. Spasticity. The current status of research and treatment. Carnforth: Parthenon Publishing, 1989;153-184.

10 Knutsson E, Martensson A, Gransberg L. Antiparetic and antispastic effects induced by tizanidine in patients with spastic paresis. 7 Neurol Sci 1982;53:187-204

11 Mathias CJ, Luckitt J, Desai P, Baker H, El Masri W, Frankel $\mathrm{H}$. Pharmacodynamics and pharmacokinetics of the oral antispastic agent tizanidine in patients with spinal cord injury. 7 Rehabil Res Dev 1989;26:9-16.

12 Wartenberg R. Pendulousness of the legs as a diagnostic test. Neurology 1951;1:18-24.

13 Bajd T, Vodovnik L. Pendulum testing of spasticity. $\mathcal{F}$ Biomed Eng 1984;6:9-16.

14 Brown RA, Lawson DA, Leslie GC, et al. Does the Wartenberg pendulum test differentiate quantitatively between spasticity and rigidity? A study in elderly stroke and Parkinsonian patients. $\mathcal{F}$ Neurol Neurosurg Psychiatry 1988;51:1178-86.

15 Ashworth B. Preliminary trial of carisoprodol on multiple sclerosis. Practioner 1964;192:540-2.

16 Abt K. Descriptive data analysis: a concept between confirmatory and exploratory data analysis. Methods of Information in Medicine 1987;26:77-8.

17 Hassan N, McLellan DL. Double-blind comparison of single doses of DS 103-282, baclofen and placebo for suppression of spasticity. $\mathcal{F}$ Neurol Neurosurg Psychiatry 1980;43:1132-6. 